\title{
Neuroinflammation and Mitochondrial Dysfunction in the Pathogenesis of Alzheimer's Disease: Modulation by Coriolus Versicolor (Yun-Zhi) Nutritional Mushroom
}

Angela Trovato' , Manuela Pennisi ${ }^{1,5}$, Rosalia Crupi ${ }^{2 *}$, Rosanna Di Paola ${ }^{2}$, Alice Alario' ${ }^{1}$, Sergio Modafferi', Gabriele Di Rosa', Tito Fernandes ${ }^{3}$, Anna Signorile ${ }^{4}$, Luigi Maiolino ${ }^{6}$, Salvatore Cuzzocrea ${ }^{2}$ and Vittorio Calabrese ${ }^{1 * *}$

'Department of Biomedical and Biotechnological Sciences, School of Medicine, University of Catania, Catania, Italy

${ }^{2}$ Department of Chemical, Biological, Pharmaceutical and Environmental Sciences, University of Messina, Messina, Italy ${ }^{3}$ Faculty of Veterinary Medicine, Lisbon University, Portugal

${ }^{4}$ Department of Basic Medical Sciences, Neurosciences and Sense Organs, University of Bari, Bari, Italy

${ }^{5}$ Spinal Unit, Emergency Hospital "Cannizzaro", Catania, Italy

${ }^{6}$ Department of Clinical and Experimental Medicine, School of Medicine, University of Catania, Catania, Italy

\section{Article Info}

\section{Article Notes}

Received: October 11, 2016

Accepted: January 23, 2017

\section{${ }^{*}$ Correspondence:}

Prof. Vittorio Calabrese

Department of Biomedical and Biotechnological Sciences,

University of Catania,

Via Andrea Doria - 95100 Catania, Italy

Phone: 0039-095-7384067; Fax: 0039-095-580138

E-mail address: calabres@unict.it

(c) 2017 Calabrese V. This article is distributed under the terms of the Creative Commons Attribution 4.0 International License

\section{Keywords}

Alzheimer's Disease

Mitochondria

Inflammasome

Nutritional Mushrooms

Pediatric

Physiopathology

Cytokines

\section{ABSTRACT}

Abnormal redox homeostasis and oxidative stress have been proposed to play a role in the etiology of several neuropsychiatric disorders and emerging interest has recently focused on markers of oxidative stress and neuroinflammation in neurodegenerative disorders as well as in different forms of chronic mental illness. Oxidative stress and altered antioxidant systems have been considered an important factor underlying the pathogenesis of Alzheimer's disease (AD). Altered expression of genes related to oxidative stress, oxidative damage to DNA, protein and lipids, as well as alterations in the redox state in central and peripheral tissues could act synergistically, and contribute to the course of the disease. Specifically, we discuss the emerging role of lipoxinA4 and inflammasome in neurodegeneration. However, the notion that low levels of stress can induce responses that may be protective against the pathogenic processes is a frontier area of neurobiological research focal to understanding and developing therapeutic approaches to neurodegenerative disorders. Herein, we discuss the potenial therapeutic role of Coriolus versicolor, a mushrooom, well known in China as Yun Zhi. We propose a potentially innovative treatment for $A D$ and, possibly, other neurodegenerative conditions associated to neuroinflammation.

\section{Introduction}

Alzheimer's disease (AD) affecting more than 45 million people worldwide is a progressive neurodegenerative disorder and represents the most common cause of dementia in the elderly, accounting for $50-60 \%$ of all cases in Western world ${ }^{1}$. The prevalence rates for $\mathrm{AD}$ rise exponentially with age, increasing markedly after 65 years. AD is characterized by cognitive decline beginning usually with impairment of episodic memory, involving progressively all cognitive functions in the late stage ${ }^{2}$. The pathological hallmarks of $\mathrm{AD}$ are amyloid plaques, containing amyloid- $\beta$ peptide, derived from the transmembrane amyloid precursor protein, and neurofibrillary tangles, composed of hyperphosforylated tau protein, in the medial temporal lobe structures and cortical areas of the brain together with neuronal death and synapses $\operatorname{loss}^{3,4}$. Brain inflammation has been linked to many of these diseases, including amyotrophic lateral sclerosis (ALS), multiple sclerosis (MS), Parkinson's disease (PD) and, particularly, Alzheimer's disease (AD) ${ }^{5}$. To adapt to environmental changes and survive different types of injuries, brain cells have evolved networks of responses that detect and control diverse forms of stress ${ }^{6,7}$. Consistent with this notion, integrated survival 


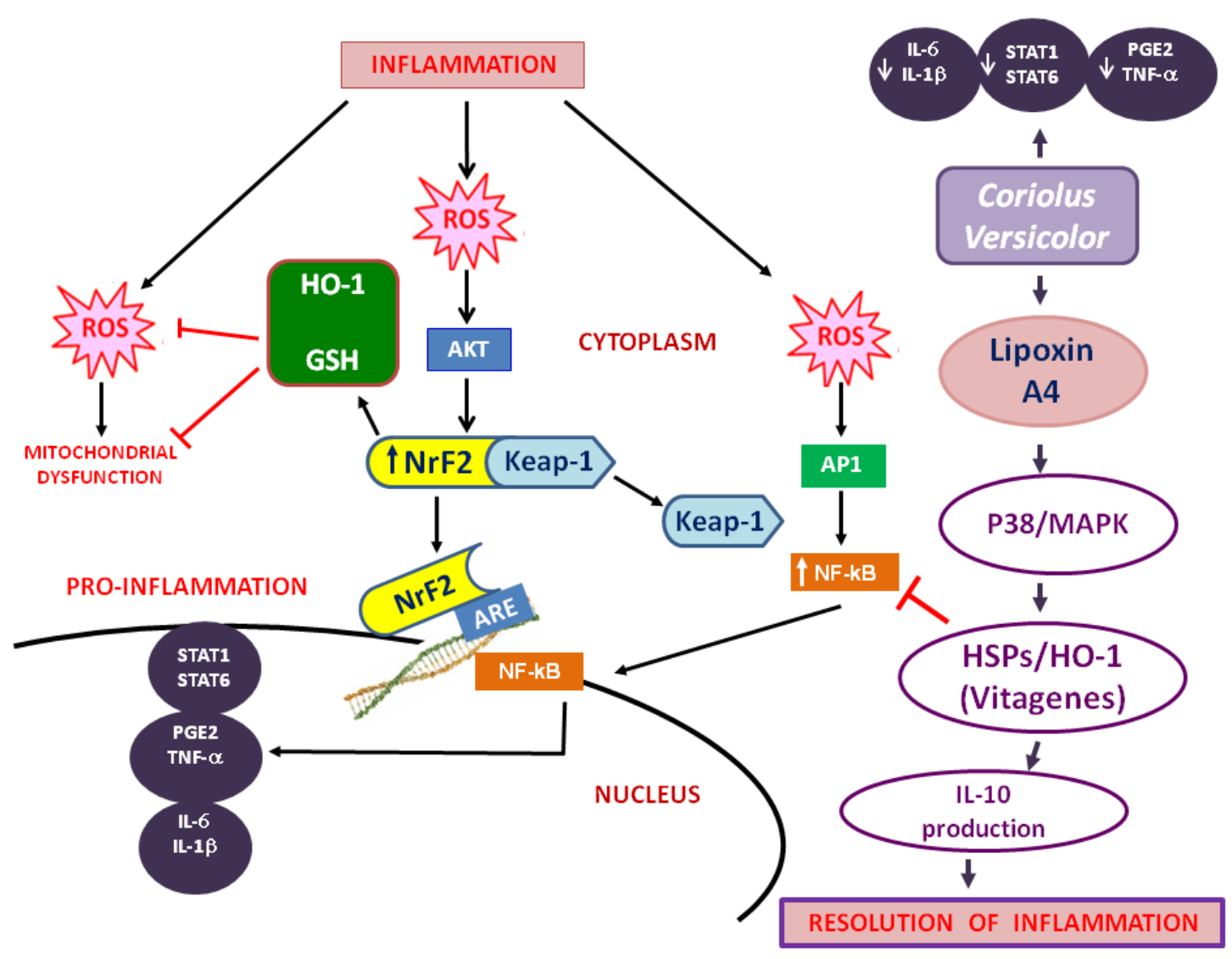

Figure 1. The pathways affected by this compound in relation to inflammation.

responses exist in the brain, which are under control of redox-dependent genes, called vitagenes (Figure 1), including heat shock proteins (Hsps), sirtuins, thioredoxin and lipoxin A4. These proteins actively operate in detecting and controlling diverse forms of stress and neuronal injuries $^{7-9}$. LXA4, a metabolic product of arachidonic acid, is considered an endogenous "stop signal" for inflammation, and shows potent anti-inflammatory properties in many inflammatory disorders, such as nephritis, periodontitis, arthritis, inflammatory bowel disease ${ }^{10}$. Microglia play an essential role in innate immunity, homeostasis, and neurotropic support in the CNS. In Alzheimer disease (AD), these cells may affect disease progression by modulating the buildup of amyloid- $\beta(A \beta)$ or by releasing proinflammatory cytokines and neurotoxic substances. Discovering agents capable of increasing Lipoxin A4 and consequently of increasing $\mathrm{Ab}$ uptake by phagocytic cells is of potential therapeutic interest for $\mathrm{AD}$. Lipoxin A4 (LXA4) as an endogenously produced eicosanoid, blocks the generations of pro-inflammatory cytokines and toxic compounds including reactive oxygen species (ROS), thereby promoting resolution of inflammation, and acts as an endogenous "braking signal" in the inflammatory process. Mushrooms, from which LXA4 is derived, have been used in traditional medicine for thousands of years. Many controlled studies have since investigated the long list of medicinal actions thought to be associated with extracts of these and other mushrooms, including antitumor, immunomodulatory, antioxidant, antiviral, antibacterial, and hepatoprotective effects. Mushrooms are a rich source of these polysaccharides, and many have been shown to stimulate host immune responses ${ }^{11}$. Indeed, the effects of polysaccharides on the immune system are by far the most exploited attribute of "medicinal mushrooms". Some of the most potent immunostimulatory molecules derived from mushrooms are the $\beta$-glucans, which activate many types of immune cells and stimulate cytokine responses. Taken all this into account and given the neuroinflammatory pathogenesis of neurodegenerative damage present in Alzheimer's disease, in our recent study we demonstrate the neuroprotective role of Coriolus versicolor biomass preparations against the inflammatory process associated to neurodegeneration evaluating also the impact on brain cellular stress response mechanisms.

Mitochondria and Neuroinflammation: Role of the Inflammasome

\section{Mitochondria and ROS}

Mitochondria have long been considered the "power- 
house of the cell" due to their primary function in energy production through oxidative phosphorylation and generation of adenosine triphosphate (ATP). The mitochondria contain two membranes, the outer (OMM) and the inner membrane (IMM) that separate two distinct compartments: the inter-membrane space and the matrix. The synthesis of ATP occurs through the respiratory electron transport chain (ETC) which is located at the inner mitochondrial membrane and consists of five protein complexes (Complexes I-V) ${ }^{12}$. Generation of ATP by mitochondria occurs via oxidative phosphorylation which requires reduction of oxygen to promote oxidation of nutrients and release of ATP. However, reduction of oxygen can generate potentially harmful intermediates such as superoxide anion radical $\left(\mathrm{O}^{2-}\right)$ and hydrogen peroxide $\left(\mathrm{H}_{2} \mathrm{O}_{2}\right)$, which can further react with other Radical Oxygen Species (ROS) or with a transition metal to form highly reactive secondary ROS, including the hydroxyl radical $(\bullet \mathrm{OH})^{13}$. There are eight sites in mitochondria that are known to possess the ability to produce $\mathrm{O}^{2-}$ [Brand, 2010]; however, their contribution to cellular ROS levels in vivo is unclear ${ }^{14}$. Interestingly, while seven of these sites can deposit $\mathrm{O}^{2-}$, into the mitochondrial matrix, only site IIIQo (on complex III) and glycerol 3-phosphate dehydrogenase can release $0^{2-}$, into the intermembrane space ${ }^{15}$.

It has been suggested that primary ROS are wellcontrolled molecules within the cell and that their reactions with target molecules can be reversible, placing this type of ROS as important intracellular signalling molecules ${ }^{16}$. In contrast, secondary ROS, particularly $\bullet \mathrm{OH}$, are catalytically very reactive, not stringently controlled within the cell and therefore comprise the main damaging type of $\operatorname{ROS}^{17,18}$. The - $\mathrm{OH}$ radical is a highly reactive specie able to react with a vast range of macromolecules such as lipids, proteins and nucleic acids. $\bullet \mathrm{OH}$ radicals are important inducers of DNA oxidation resulting in DNA lesions that often include single oxidised nucleobases, tandem base modifications, intra- and interstrand cross-links, oligonucleotide singlestrand breaks and 2- deoxyribose oxidation products ${ }^{17}$. Furthermore, during lipid peroxidation, allylically activated $\mathrm{CH}_{2}$ groups present in lipids, most often in polyunsaturated fatty acids (PUFAs), are especially prone to hydrogen abstraction by $\bullet \mathrm{OH}$ radicals resulting in the oxidation of these lipids in the cell ${ }^{19}$. The reaction of ROS with proteins can involve irreversible modifications producing protein carbonyl groups. In the case of proteins rich in cysteine and methionine residues, both of which contain susceptible sulfur atoms, are also particularly prone to oxidative damage. Cysteine oxidation leads to the formation of disulfide bonds, mixed disulfides, and thiyl radicals, while methionine sulfoxide is the major product of methionine oxidation $^{20}$. The oxidation of cysteine and methionine is reversible as cells are equipped with systems, such as methoninesulfoxide reductase, glutathione, and thioredoxin redox system, capable of reversing the oxidation ${ }^{21}$. Each of these modifications can change the activity of the target protein, thereby altering its function in a signaling pathway. Phosphatases appear to be susceptible to regulation by ROS in this manner, as they possess a reactive cysteine their catalytic domain that can be reversibly oxidized, which inhibits their dephosphorylation activity ${ }^{22}$. Specific examples of phosphatases known to be regulated in this manner are PTP1b, PTEN, and MAPK phosphatases ${ }^{23}$.

\section{Redox homeostasis}

The action of ROS is usually balanced by the antioxidative capacity of a cell or organism and a disturbance of this balance in favor of a prooxidant state is commonly referred to as oxidative stress. The most well-known endogenous mitochondrial antioxidant enzyme is superoxide dismutase (SOD) which convert $\mathrm{O}_{2}$ to $\mathrm{H}_{2} \mathrm{O}_{2}$. SOD1 is located in the mitochondrial intermembrane space and cytosol, SOD2 is located in the mitochondrial matrix, and SOD3 is tethered to the extracellular matrix. Other antioxidant enzymes that remove $\mathrm{H}_{2} \mathrm{O}_{2}$ are peroxiredoxins (PRXs), glutathione peroxidases (GPXs), and catalase. Mammalian cells express 6 PRX isoforms, including PRX3 and PRX5 in the mitochondria. PRXs are enzymes that function by undergoing oxidation by $\mathrm{H}_{2} \mathrm{O}_{2}$ at an active site cysteine and then subsequent reduction by thioredoxin, thioredoxin reductase, and $\mathrm{NADPH}^{24}$. There are eight isoform of GPXs, which are oxidized by $\mathrm{H}_{2} \mathrm{O}_{2}$ and reduced by glutathione (GSH). Catalase is found in peroxisomes. Function of these antioxidant enzymes is dependent on how fast they react with $\mathrm{H}_{2} \mathrm{O}_{2}$ (rate constant, $\mathrm{k}$ ) and the concentration of $\mathrm{H}_{2} \mathrm{O}_{2}$ and enzyme in vivo. Importantly, in vivo concentrations of $\mathrm{H}_{2} \mathrm{O}_{2}$ are not well defined. Nevertheless, peroxiredoxins have a high rate constant and high abundance and therefore are thought to be responsible for scavenging nanomolar levels of $\mathrm{H}_{2} \mathrm{O}_{2}$ associated with signaling.

GPXs have similar rate constants, but are less abundant and therefore are likely only important at higher intracellular concentrations of $\mathrm{H}_{2} \mathrm{O}_{2}$ when GPXs can begin to compete with PRXs for substrate ${ }^{25}$. Therefore, it is possible that PRXs are critical for turning ROS signaling off, while GPXs are critical for buffering high levels of ROS to bring them to a level at which the cell evades damage and can initiate signaling stress responses. Catalase has an even lower affinity for hydrogen peroxide. Regulation of activity and expression levels of these antioxidants occurs by a variety of mechanisms and functions in part to manage ROS levels.

The mitochondrial free radical theory of aging postulates that the damage caused by accumulating ROS produced by mitochondria is the driving force behind aging $^{26}$. This theory is corroborated to some extent by the inverse correlation between mitochondrial ROS production and lifespan in mammals ${ }^{27}$. 
Current evidence points to mitochondrial dysfunction as an overarching mechanism of aging and age-related disease. It is implicated in an extensive list of aging pathologies such as cancer, intestinal barrier dysfunction, depression, chronic obstructive pulmonary disease (COPD), diabetes, and others ${ }^{28-30}$.

ROS are not the only aspect of flawed mitochondria that contributes to degenerating health, the emerging picture is that mitochondrial dysfunction in human aging and aging-associated diseases are not limited to accumulation of mtDNA mutations, but extend to abnormalities in mitochondria biogenesis, turnover, dynamics, and proteostasis. Mitochondria undergo constant fusion and fission to maintain a balance between mitochondrial biogenesis and mitochondrial autophagy (mitophagy) or apoptosis ${ }^{31}$. Several of the proteins involved in mitochondrial dynamics have been well characterized. Mitofusin 1 (Mfn1), mitofusin 2 (Mfn2) and optic atrophy-1 (Opa1) are the major proteins involved in the mitochondrial fusion process. Dynamin related protein-1 (Drp1) and fission protein 1 (Fis1) are involved in the fission process. The fusion process is activated during conditions of increased mitochondrial bioenergetics. The fission process is activated during mitochondrial degradation through the autophagosome (autophagy) ${ }^{32}$. The nuclear protein, peroxisome proliferator-activated receptor- $\gamma$ coactivator- $1 \alpha$ (PGC- $1 \alpha)$, is a key mediator of mitochondrial biogenesis and an inducer of Mfn2. Perturbations of the IMM and OMM during ROS leakage are exacerbated by disrupted fusion and fission regulatory pathways. Thus, a deleterious feedback loop between ROS and dynamics leads to mitochondrial ROS dysfunction and subsequent apoptosis. Mitochondrial Sirtuins are modulators of energy metabolism, DNA repair and oxidative stress. Sirt- 1 is both a nuclear and cytoplasmic protein and has been observed in mitochon- dria, while Sirt-3, 4, and 5 are mitochondrial proteins $^{33}$.

In relation to the redox status, Sirt- 3 increases the transcription factor, Forkhead box 03 (FOXO3), binding to the mitochondrial superoxide scavenger genes SOD2/ MnSOD, cytochrome C oxidase assembly protein (SCO2), and catalase ${ }^{34}$, thus modulating oxidative stress. Sirt-3 can also directly activate SOD2 activity by deacetylating $\mathrm{it}^{35}$.

Interestingly, brain-specific overexpression of SIRT1 results in long-lived animals which, when they age, seem to have preserved mitochondrial morphology along with increased oxygen consumption and more robust physical activity $^{36}$. Similarly, whole-body overexpression of SIRT6 can result in lifespan extension for male mice ${ }^{37}$. The sirtuin family members that localize to the mitochondria (SIRT3, SIRT4, and SIRT5) have widespread metabolic effects, including regulating oxidative phosphorylation ${ }^{38}$, altering fatty acid oxidation ${ }^{39,35,40}$.
A vast body of data has accumulated linking mitochondrial redox metabolism to the aging process. Similarly, a growing number of dietary interventions have been demonstrated to modulate mitochondrial ROS production, detoxification and oxidative damage repair. Many (but not all) of these dietary interventions are associated with lifespan extension, or protection against age-related disease, in mammals. The term "nutraceutical" is actually a combined form of "nutrition" and "pharmaceutical." The generally accepted definition is "a food or part of a food which provides health benefits, including the prevention and/or treatment of a disease." Most nutraceuticals are dietary supplements. Studies both in vitro and in vivo reveal that consumption of nutraceuticals, especially the ones with high antioxidant capacity, has an inverse relationship with cardiovascular diseases, various cancers, and diabetes. On the basis of free radical theory of ageing it is postulated that any substance with a great antioxidant capacity can be a potential candidate for delaying the aging, but more evidences, show that these substances could improve mitochondrial functions also through activation of different signaling pathways ${ }^{41,42}$. There has been a recent upsurge of interest in complementary and alternative medicine especially dietary supplements and functional foods in delaying the onset of age associated neurodegenerative disease. Different dietary interventions and nutraceuticals have been demonstrated to have beneficial effects on mitochondrial functionality in different physiopathological conditions ${ }^{43}$. Resveratrol, a natural polyphenolic compound of red wine proved to improve mitochondrial dysfunction and oxidative stress associated to Parkinson's disease (PD), through the activation of several metabolic sensors resulting in PGC- $1 \alpha$ activation ${ }^{44}$. Epigallocatechin-3-gallate (EGCG) - a natural polyphenol component of green tea counteracts the mitochondrial energy deficit and oxidative stress found in Down's syndrome (DS) cells by promotion PKA activity ${ }^{45}$. Furthermore, Hydroxytyrosol a phenol of olive oil, prevented the decline in the expression of the PGC- $1 \alpha$ transcription cascade of OXPHOS complexes through activation of PKA and CREB phosphorylation ${ }^{46}$. Interestingly, all these compounds would exert anticancer effect activating the mitochondrial apoptotic pathway ${ }^{47-49}$. The therapeutic potential of nutritional mushrooms against human leukemia is demonstrate in U937 human monocytic leukemia cells in which promote apoptosis by increase of pro-apoptotic protein ${ }^{50}$. Pharmacological manipulation of cellular stress pathways is emerging as a viable approach to treating certain neurologic diseases, such as Alzheimer's disease and psychiatric disorders, such as schizophrenia ${ }^{51}$. Specifically, small redox active molecules, such as the phytochemicals sulforaphane and hydroxytyrosol, or nutritional mushrooms may be potential candidates to modulate physiological pathways associated with cellular stress response and vitagene networks ${ }^{52}$ redox 
imbalance/oxidative stress $^{53}$, mitochondrial function ${ }^{54}$ immune response and anti-neuroinflammation ${ }^{55}$ heat shock response control $^{56}$ and synaptic dysfunction ${ }^{57}$. Conceivably, such therapeutic tactics may be of value in mitigating or perhaps preventing signs and symptoms of neurodegeneration. Increased oxidative stress has been implicated as a contributory factor to AD pathogenesis, and post-mortem studies have demonstrated increased protein carbonylation, nitration, cysteine-oxidation, lipid peroxidation, and DNA/RNA oxidation in brain and peripheral tissue samples from patients with $\mathrm{AD}$. Increased oxidative stress can damage mitochondrial proteins. Of particular relevance to the pathophysiology of major neurodegenerative disorders are findings of decreased levels ofmRNA (and protein subunits) that are involved in the transfer of electrons in complex I of the ETC, in AD patients. Decreased efficiency of the electron transfer process within complex I and complex IV, results in increased leakage and mono-electronic reduction of molecular oxygen to form the superoxide anion ${ }^{58}$, with ensuing damage to proteins, lipids and DNA. As well, an increasing body of evidence supports a role of immune activation as a prominent causative factor in the pathogenesis of a number of major neurologic and neuropsychiatric disorders ${ }^{59}$.

\section{Inflammasome}

Recent studies have demonstrated that the inflammasome modulates neuroinflammatory processes at the initial stage, with a secondary cascade of events inclusive of oxidative stress, having been shown to possess the ability to activate the inflammasome ${ }^{60}$. The inflammasome is a macromolecular complex that contains multiple copies of a receptor for pathogen- or damagederived molecular patterns (PAMPs, pro-caspase-1, and an adaptor, apoptotic speck-containing protein with a CARD [ASC], which induces caspase-1 maturation ${ }^{61}$ Active caspase- 1 is responsible for rapid, lytic cell death (pyroptosis). Upon sensing PAMP or DAMP, AIM2 and/or NLRP3, inflammasomes activate caspase-8 and caspase-1, respectively, leading to both apoptotic and pyroptotic cell death $^{61}$. The AIM2 inflammasome is activated by cytosolic DNA, and in addition, it has recently been demonstrated that mitochondria represent major sources of DAMPs capable of triggering neuroinflammatory responses, with resulting apoptosis, pyroptosis and autophagy ${ }^{62}$. Recognition of DNA by immunocompetent cells is an important immunological signature that marks the initiation of an innate immune response. AIM2 is a cytoplasmic sensor that recognizes dsDNA of microbial or host origin. Upon binding to DNA, AIM2 assembles inflammasomal multiprotein complex, which induces pyroptosis and proteolytic cleavage of the proinflammatory cytokines pro-IL-1 $\beta$ and pro-IL-18. Release of microbial DNA into the cytoplasm during infections activates the AIM2 inflammasome. For instance, inappropriate recognition of cytoplasmic self-DNA by AIM2 contributes to the development of a number of autoimmune and inflammatory diseases, as well as in neurodegenerative disorders ${ }^{63,64}$. In this scenario the mushrooms offer great potential as a polypharmaceutic drug because of the complexity of their chemical contents and different varieties of bioactivities. Available evidence suggests that mushrooms exhibit antioxidant, antitumor, antivirus, anticancer, antiinflammatory, immune modulating, anti-microbial, and antidiabetic activities ${ }^{65}$. In rat model diabetes mellitus the administration of medicinal mushrooms increases activity of antioxidant enzymes and reduces the amount of thiobarbituric acid reactive substances (TBARS) thus indicating pronounced antioxidant properties of studied mushrooms ${ }^{66}$. Also recently, it has also been shown that some mushroom extracts can produce direct cytotoxic effect on cancer cells through the action on the mitochondrial apoptotic pathway ${ }^{67}$. In contrast to plant herbal medicines, which are widely explored and relatively more advanced, the brain and cognition health effects of mushrooms are in the early stages of research. Palmitic, oleic, and linoleic acids dominate fatty acid profiles in mushrooms ${ }^{68}$. These fatty acids are important nutritionally, as oleic acid (C18:1 $\mathrm{n}-9)$ has been shown to activate SIRT1-PGC1 $\alpha$ complex $^{69}$ and promote axon generation in the striatum during brain development ${ }^{70}$. Furthermore, in vitro toxicology assessment across different mushroom extracts on embryonic fibroblast and neuroblastoma cell lines suggest that the extracts are safe to be consumed even at high doses and they may be developed as a dietary supplement to improve brain and cognitive health. It is generally recognized that inflammatory process produces large amounts of inflammatory mediators, such as cytokines, chemokines, and prostaglandins. These mediators activate multiple signal transduction cascades and transcription factors associated with inflammation involved in the development of the common pathway of inflammation, such as NF- $\kappa B$, MAPK and JAK-STAT signaling pathways, or otherwise regulating the antiinflammatory process, such as the Keap1 and Nrf2 system which, by modulating gene expression through the antioxidant response element (ARE), inhibit progression of inflammation.

\section{Extracts and bioactive compounds isolated from Coriolus versicolor (Yun-Zhi)}

Mushrooms have been used in traditional medicine for thousands of years ${ }^{71,72}$. Many controlled studies have since investigated the long list of medicinal actions thought to be associated with extracts of these and other mushrooms, including antitumor, immunomodulatory, antioxidant, antiviral, antibacterial, and hepatoprotective effects ${ }^{73}$. Mushrooms are a rich source of these polysaccharides, and many have been shown to stimulate host immune 


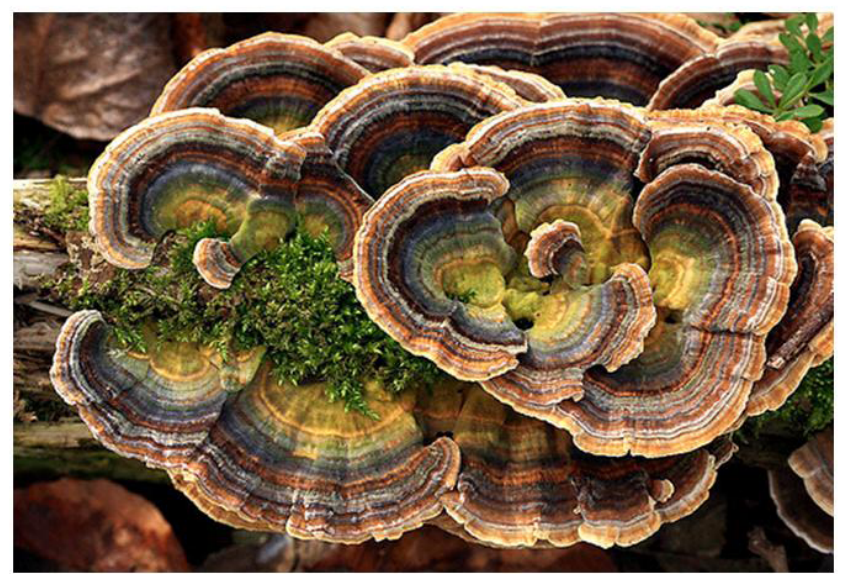

Figure 2. Coriolus versicolor mushrooms.

responses $^{74}$. Indeed, the effects of polysaccharides on the immune system are by far the most exploited attribute of MMs. Some of the most potent immunostimulatory molecules derived from mushrooms are the $\beta$-glucans, which activate many types of immune cells and stimulate cytokine responses ${ }^{75-77}$. Studies in rodents and humans suggest that polysaccharides derived from mushrooms can stimulate the immune system to attack malignant cells $^{78,79}$ and several of these compounds are now used in East countries in association to radio and chemotherapy ${ }^{80}$. In addition to the previous bioactive compounds, antiinflammatory peptides of different molecular weights have been isolated from mushrooms ${ }^{81}$. Cordymin, a low molecular weight peptide $(10,906 \mathrm{Da})$, has been purified from the medicinal mushroom Cordyceps sinensis ${ }^{82}$ and from Cordyceps militaris ${ }^{83}$. This peptide significantly inhibited the infiltration of polymorphonuclear cells and IR-induced upregulation of C3 protein produced in the brain, interleukin- $1 \beta$, and tumour necrosis factor- $\alpha$, which had a neuroprotective effect on the ischemic brain, due to the inhibition of inflammation ${ }^{71}$.

The administration of complex mixtures of molecules of unknown concentrations is difficult to reconcile with current pharmaceutical practices involving highly purified compounds. The active ingredients may be unknown, making mushroom extracts very difficult to patent. Moreover, mushroom-derived polysaccharides are complex molecules that cannot be synthesized, as the mass production of these compounds would require timely and costly extraction processes. As a result, many research efforts have focused on low molecular weight compounds, such as cordycepin, which is a cytotoxic nucleoside analog inhibitor of cell proliferation. Modern clinical practice in Asian countries continues to rely on mushroom-derived preparations. Medicinal effects have been demonstrated for many traditionally used mushrooms, including extracts of Agaricus campestris, Pleurotus ostreatus and Coriolus versicolor $^{84}$. This last is better known in Chinese landscape as Yun Zhi.

The active principle derived from Coriolus versicolor (Figure 2) belongs to a new class of elements called biological response modifiers $(\mathrm{BRM})^{85}$, which are defined as agents capable of stimulating the immune system with various therapeutic effects.

Of the mushroom-derived therapeutics, polysaccharopeptides obtained from Coriolus versicolor are commercially the best established. In addition to its medical applications, Coriolus versicolor is widely used to degrade recalcitrant organic pollutants such as pentachlorophenol $(\mathrm{PCP})^{86}$. Taken all this into account and given the neuroinflammatory pathogenesis of neurodegenerative damage present in Alzheimer's disease, present studies are undertaken to gain insight into the possible neuroprotective role of Coriolus biomass preparation against the inflammatory process and to evaluate the impact of this intervention on cellular stress response mechanism operating in the central nervous system ${ }^{11,87}$. Of particular interest are the polysaccharopeptides produced by Coriolus versicolor which are used to supplement the chemotherapy and radiotherapy of cancer and infectious diseases. The best known commercial polysaccharopeptide preparations of Coriolus versicolor are Polysaccharide K or Krestin (PSK) and polysaccharopeptide (PSP). Both products are obtained from the extraction of Coriolus versicolor mycelia, PSK is obtained from the extraction of Coriolus versicolor (CM-101) strains, while PSP obtained from the extraction of Coriolus versicolor (Cov-1) strains ${ }^{88,89}$; PSP and PSK are chemically similar: $(1,3) \beta$-glucan branched at $4^{\prime}$ and $6^{\prime}$ positions and they differ mainly in the presence of fucose in PSK and rhamnose and arabinose in PSP (Figure 3). PSP and PSK are powders that are soluble and stable in hot water. These polysaccharopeptides have similar physiological activities. An extremely broad range of physiological effects has been linked with the use of Coriolus versicolor polysaccharopeptides. Some of the main effects include the following: immunopotentiation by inducing production of interleukin-6, interferons, immunoglobulin-G, macrophages, and T-lymphocytes; counter immunosuppressive effects of chemotherapy, radiotherapy, and blood transfusion; antagonization of immunosuppression induced by tumors; inhibition of proliferation of various cancers by inducing production of superoxide dismutase (SOD), glutathione peroxidase (Figure 4), and general immune enhancement; improvement of appetite and liver function; calming of the central nervous system ${ }^{86}$. Superoxide dismutase is an important enzyme that scavenges oxygen free radicals and the SOD plays an important role in the response to stress to counteract the toxicity of active oxygen. In particular, PSK could PSK could enhance SOD activity and increase the contents of MnSOD mRNA in mouse peritoneal macrophages $^{90}$. PSP/PSK possess anticancer activity ${ }^{91,92}$. 


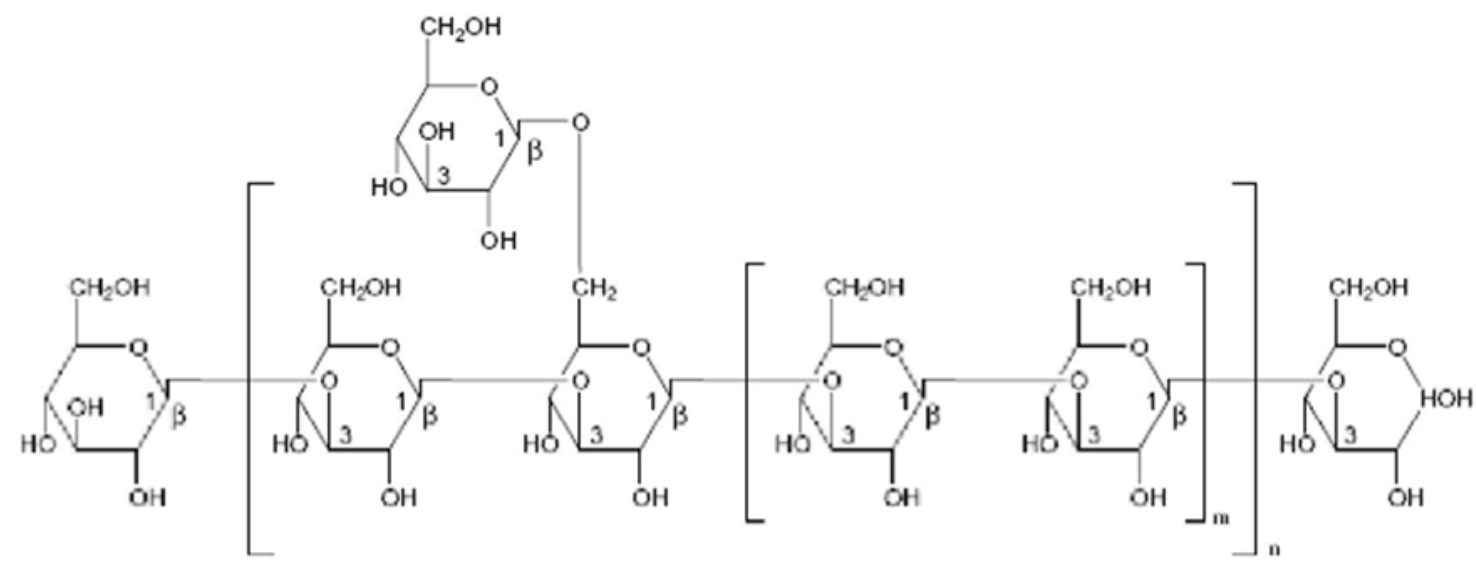

Figure 3. Typical structures of polysaccharide portions of the polysaccharide peptide (PSP) of COV-1 strain of Coriolus versicolor (Zhou and Yang, 1999).

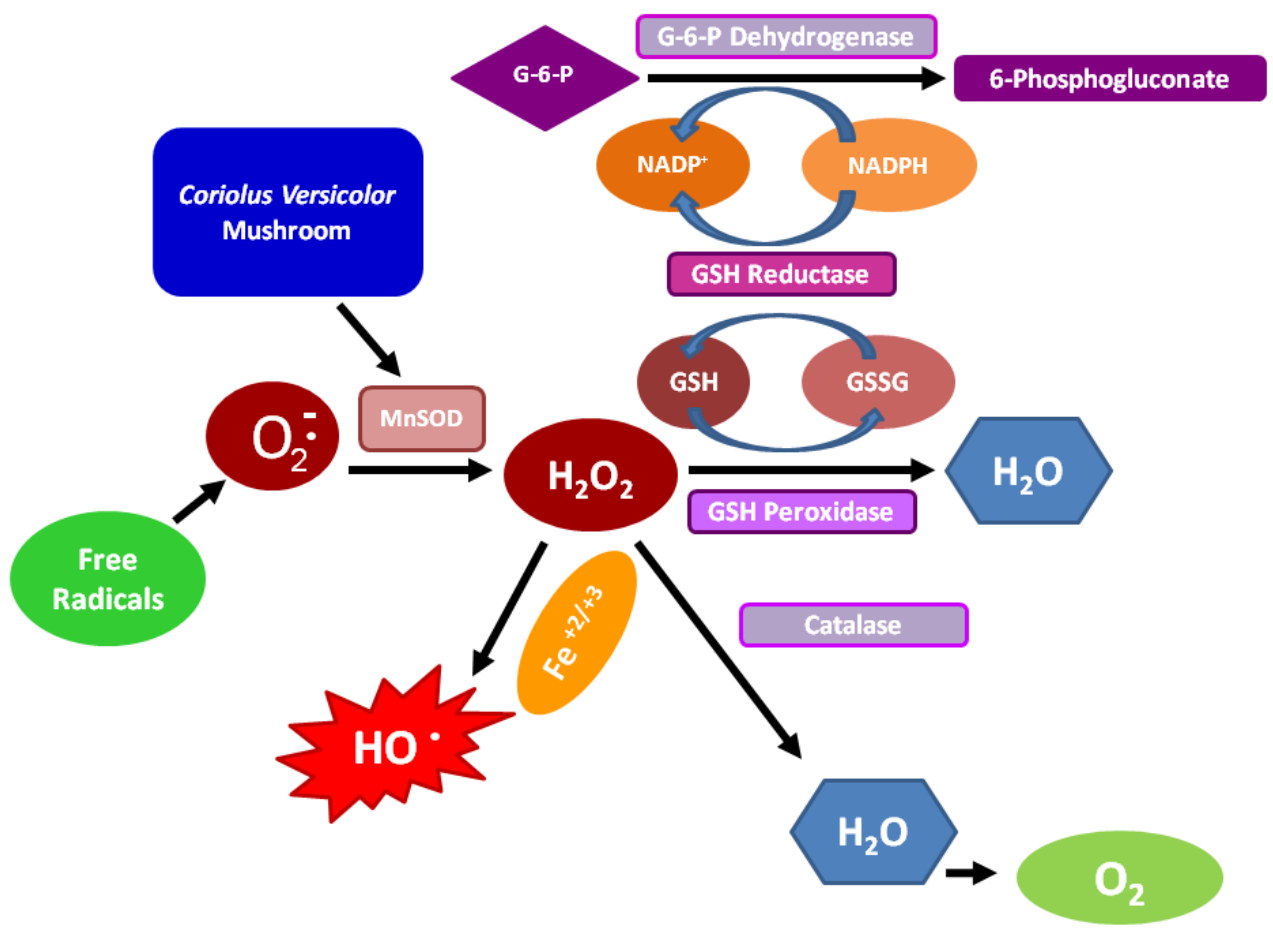

Figure 4. The redox pathway and the relation with mushroom compounds.

Oral administration of PSK/PSP has controlled various carcinomas in experimental animals and humans ${ }^{93}$. It is reported that PSK induces apoptosis in HL-60 cells through the activation of mitochondrial and caspase-dependent pathways involving activation of p38 MAPK signaling cascades $^{94}$ and overexpression of proapoptotic protein $\mathrm{Bax}^{95}$.

\section{Conclusions}

Accumulating evidence have indicated that oxidative stress and ROS play an important role in the progression of many chronic diseases including cardiovascular diseases, diabetes, and neurodegenerative disorders ${ }^{96}$ [Nakamura et al., 2012]. Imbalance between ROS generation and antioxidant enzyme activities will cause lipid peroxidation, nuclear and mitochondrial DNA damage and protein oxidation, resulting in brain damage and amnesia ${ }^{97}$ [Biasibetti et al., 2013]. A growing number of dietary interventions have been demonstrated to modulate mitochondrial ROS production, detoxification 
and oxidative damage repair. Many (but not all) of these dietary interventions are associated with lifespan extension, or protection against age-related disease, in mammals. Emerging nutraceuticals are showing promise as modulators of mitochondrial redox metabolism capable of eliciting beneficial outcomes. Mushrooms, known for their potent antioxidant property, have attracted interest due to their potential in neuroprotection, antioxidant, and anti-inflammatory effects, in mitochondrial dysfunctions associated disorders.

\section{References}

1. Ballard C, Gauthier S, Corbett A, et al. Alzheimer's disease. Lancet 2011; 19: 1019-1031.

2. Mayeux R, Stern Y. Epidemiology of Alzheimer disease. Cold Spring Harb Perspect Med. 2012; 1: 2-8.

3. Gandy S. The role of cerebral amyloid beta accumulation in common forms of Alzheimer disease. J Clin Invest. 2005; 115: 1121-1129.

4. Iqbal K, Alonso Adel C, Chen S, et al. Tau pathology in Alzheimer disease and other tauopathies. Biochim Biophys Acta. 2005; 3 1739(2-3): 198-210.

5. Amor S, Puentes F, Baker D, et al. Inflammation in neurodegenerative diseases.Immunology. 2010; 129(2): 154-169.

6. Calabrese V, Cornelius C, Dinkova-Kostova AT, et al. Biochim Cellular stress responses hormetic phytochemicals and vitagenes in aging and longevity. Biophys Acta. 2012 May; 1822(5): 753-83.

7. Dattilo S, Mancuso C, Koverech G, et al. Heat shock proteins and hormesis in the diagnosis and treatment of neurodegenerative diseases. Immun Ageing. 2015; 12: 20.

8. Calabrese V, Stella AM, Butterfield DA, et al. Redox regulation in neurodegeneration and longevity role of the heme oxygenase and HSP70 systems in brain stress tolerance.Antioxid Redox Signal. 2004 Oct.

9. Arnèr ESJ, Holmgren A. Physiological functions of thioredoxin and thioredoxin reductase. Eur J Biochem. 2000; 267: 6102-6109.

10. Romano M, Cianci E, Simiele F, et al. Lipoxins and aspirin-triggered lipoxins in resolution of inflammation. Eur J Pharmacol. 2015 Aug 5; 760: 49-63.

11. Trovato A, Siracusa R, Di Paola R, et al. Redox modulation of cellular stress response and lipoxin A4 expression by Coriolus versicolor in rat brain Relevance to Alzheimer's disease pathogenesis. Neurotoxicology. 2016a; 53: 350-358.

12. McBride $H$, Neuspiel M, Wasiak S. Mitochondria more than just a powerhouseCurr. Biol. 2006; 16: 60.

13. Murphy MP. How mitochondria produce reactive oxygen species. Biochem J. 2009; 417: 1-13.

14. Brand MD. The sites and topology of mitochondrial superoxide production. Exp. 2010.

15. Sena LA, Chandel NS. Physiological roles of mitochondrial reactive oxygen species. Mol Cell. 2012 Oct 26; 48(2): 158-67. doi: 10.1016/j. molcel.2012.09.025

16. Daiber A. Redox signaling cross-talk from and to mitochondria involves mitochondrial pores and reactive oxygen species Biochim. Biophys Acta. 2010; 1797: 897-906.

17. Cadet J, Wagner JR. Oxidatively generated base damage to cellular DNA by hydroxyl radical and one-electron oxidants similarities and differences. Arch Biochem Biophys. 2014; 557: 47-54.
18. Weidinger A, Kozlov AV. Biological activities of reactive oxygen and nitrogen species oxidative stress versus signal transduction. Biomolecules. 2015; 5: 472-484.

19. Spiteller G. Lipid peroxidation in aging and age-dependent diseases. Exp Gerontol. 2001; 36: 1425-1457.

20. Finkel T. From sulfenylation to sulfhydration what a thiolate needs to tolerate. Sci Signal. 13 Mar 2012

21. Celi P, Gabai G. Oxidant/Antioxidant Balance in Animal Nutrition and Health The Role of Protein Oxidation. Front Vet Sci. 2015 Oct 26; 2: 48.

22. Rhee SG, Bae YS, Lee SR, et al. Hydrogen peroxide a key messenger that modulates protein phosphorylation through cysteine oxidation. Sci STKE. 2000 Oct 10; 53: pe1. Review.

23. Tonks NK. Redox redux revisiting PTPs and the control of cell signaling. Cells June 2005.

24. Cox AG, Winterbourn CC, Hampton MB. Mitochondrial peroxiredoxin involvement in antioxidant defence and redox signalling.Biochem J. 2009 Dec 23; 425(2): 313-25. Review.

25. Winterbourn CC, Hampton MB. Thiol chemistry and specificity in redox signaling. Free Radic Biol Med. 2008 Sep 1; 45(5): 549-61.

26. Harman D. The biologic clock: the mitochondria. J Am Geriatr Soc. 1972; 20: 145-147.

27. Ku HH, Brunk UT, Sohal RS. Relationship between mitochondrial superoxide and hydrogen peroxide production and longevity of mammalian species. Free Radic Biol Med. 1993; 15: 621-627.

28. Anderson G, Maes M. Oxidative/nitrosative stress and immunoinflammatory pathways in depression treatment implications Curr. Pharm Des. 2014; 20: 3812-3847.

29. Boland ML, Chourasia AH, Macleod KF. Mitochondrial dysfunction in cancer. Front Oncol. 2013; 3: 292.

30. Montgomery MK, Turner N. Mitochondrial dysfunction and insulin resistance an update. Endocr Connect. 2015; 4: R1-R15.

31. Archer SL. Mitochondrial dynamics mitochondrial fission and fusion in human diseases. N Engl J Med. 2013; 369: 2236-2251.

32. Westermann B. Bioenergetic role of mitochondrial fusion and fission. Biochim Biophys Acta. 2012; 1817: 1833-1838.

33. Parihar P, Solanki I, Mansuri ML, et al. Mitochondrial sirtuins emerging roles in metabolic regulations energy homeostasis and diseases. Exp Gerontol. 2015 Jan; 61: 130-41.

34. Verdin E, Hirschey MD, Finley LW, et al. Sirtuin regulation of mitochondria energy production apoptosis and signaling. Trends Biochem Sci. 2010; 35: 669-675.

35. Qiu X, Brown K, Hirschey MD, et al. Calorie restriction reduces oxidative stress by SIRT3-mediated SOD2 activation. Cell Metab. 2010; 12: 662-667.

36. Satoh A. Sirt1 extends life span and delays aging in mice through the regulation of Nk2 homeobox 1 in the DMH and LH. Cell Metab. 2013; 18: $416-430$

37. Kanfi Y. The sirtuin SIRT6 regulates lifespan in male mice. Nature. 2012; 483: 218-221.

38. Ahn BH, Kim HS, Song S, et al. A role for the mitochondrial deacetylase Sirt3 in regulating energy homeostasis. Proc Natl Acad Sci USA. 2008; 105: $14447-14452$.

39. Hirschey MD. SIRT3 regulates mitochondrial fatty-acid oxidation by reversible enzyme deacetylation. Nature. 2010; 464: 121-125.

40. Tao R, Coleman MC, Pennington D, Ozden O, Park SH.

41. Ahmad A, Ginnebaugh KR, Li Y, et al. Molecular targets of naturopathy in cancer research bridge to modern medicine. Nutrients. 2015 Jan 6; $7(1): 321-34$. 
42. Tan HK, Moad AI, Tan ML. The mTOR signalling pathway in cancer and the potential mTOR inhibitory activities of natural phytochemicals. Asian Pac J Cancer2014;15(16):6463-75.

43. Page MM, Robb EL, Salway KD, et al. Mitochondrial redox metabolism aging longevity and dietary effects. Mech Ageing Dev. 2010 Apr; 131(4): 242-52.

44. Ferretta A, Gaballo A, Tanzarella P, et al. Effect of resveratrol on mitochondrial function implications in parkin-associated familiar Parkinson's disease. Biochim Biophys Acta. 2014 Jul; 1842(7): 90215

45. Valenti D, De Rasmo D, Signorile A, et al. Epigallocatechin-3gallate prevents oxidative phosphorylation deficit and promotes mitochondrial biogenesis in human cells from subjects with Down's syndrome. Biochim Biophys Acta. 2013 Apr; 1832(4): 542-52.

46. Signorile A, Micelli L, De Rasmo D, Santeramo A, Papa F, Ficarella R, et al. Regulation of the biogenesis of OXPHOS complexes in cell transition from replicating to quiescent state: involvement of PKA and effect of hydroxytyrosol. Biochim Biophys Acta. 2014; 1843(4):675-84.

47. Gorlach S, Fichna J, Lewandowska U. Polyphenols as mitochondriatargeted anticancer drugs. Cancer Lett. 2015 Oct 1; 366(2): 141-9.

48. Oliveira MR, Nabavi SF, Daglia M, et al. Epigallocatechin gallate and mitochondria-A story of life and death. Pharmacol Res. 2016 Feb; 104 70-85.

49. de Oliveira MR, Nabavi SF, Manayi A, et al. Resveratrol and the mitochondria From triggering the intrinsic apoptotic pathway to inducing mitochondrial biogenesis a mechanistic view.Biochim Biophys Acta. 2016 Apr; 1860(4): 727-45.

50. Kim SP, Kang MY, Choi YH, et al. Mechanism of Hericium erinaceus Yamabushitake mushroom-induced apoptosis of U937 human monocytic leukemia cells. Food Funct. 2011 Jun; 2(6): 348-56.

51. Ghosh N, Ghosh R, Mandal SC. Antioxidant protection A promising therapeutic intervention in neurodegenerative disease. Free Radic Res. 2011 Aug; 45(8): 888-905.

52. Sarsour EH, Maneesh G, Kumar G, et al. MnSOD activity regulates hydroxytyrosol-induced extension of chronological. lifespan Age. 2012 Feb; 34(1): 95-109. Published online 2011 Mar 8

53. Mihailović M, Jovanović J A, Uskoković A, et al. Protective Effects of the Mushroom Lactarius deterrimus Extract on Systemic Oxidative Stress and Pancreatic Islets in Streptozotocin-Induced Diabetic Rats. J Diabetes Res. 2015.

54. Greco T, Shafer J, Fiskum G. Sulforaphane Inhibits Mitochondrial Permeability Transition and Oxidative Stress Free Radic. Biol Med. Published online 2011 Sep 21

55. Millington C, Sonego S, Karunaweera N, et al. Chronic Neuroinflammation in Alzheimer's Disease New Perspectives on Animal Models and Promising Candidate Drugs. Biomed Res Int. 2014.

56. Phan CW, Pamela David P, Tan YS, et al. Intrastrain Comparison of the Chemical Composition and Antioxidant Activity of an Edible Mushroom Pleurotus giganteus and Its Potent Neuritogenic Properties. ScientificWorldJournal. 2014.

57. Singh K, Connors SL, Macklin EA, Smith KD, Fahey JW, Talalay P, Zimmerman AW. Sulforaphane treatment of autism spectrum disorder (ASD). Proc Natl Acad Sci U S A. 2014 Oct 28;111(43):15550-5.

58. Giachin G, Bouverot R, Acajjaoui S, et al. Dynamics of Human MitochondrialComplexI Assembly Implications for Neurodegenerative Diseases.Front Mol Biosci. 2016 Aug 22; 3: 43.

59. Kurt M. Lucin and Tony Wyss-Coray Neuron Immune activation in brain aging and neurodegeneration too much or too little. 2009 Oct 15; 64(1): 110-122.
60. Kim YK, Na KS, Myint AM, et al. The role of pro-inflammatory cytokines in neuroinflammation neurogenesis and the neuroendocrine system in major depression. Prog Neuropsychopharmacol Biol Psychiatry. 2016 Jan 4; 64: 277-84.

61. He Y, Hara H, Núñez G. Mechanism and Regulation of NLRP3 Inflammasome Activation. Trends Biochem Sci. 2016 Sep 23.

62. Cherry AD, Piantadosi CA. Regulation of mitochondrial biogenesis and its intersection with inflammatory responses. Antioxid Redox Signal. 2015 Apr 20; 22(12): 965-76.

63. Alnemri ES. Sensing cytoplasmic danger signals by the inflammasome. J Clin Immunol. 2010 Jul; 30(4): 512-9.

64. Smith S, Jefferies C. Role of DNA/RNA sensors and contribution to autoimmunity. Cytokine Growth Factor Rev. 2014 Dec; 25(6): 745-57.

65. Roupas p, Keogh J, Noakes M. To role of edible mushrooms in health: evaluation of the evidence. J. Funct foods. 2012; 4: 687-709.

66. Yurkiv B, Wasser P, Nevo E, et al. Antioxidant Effects of medicinal Mushrooms Agaricus brasiliensis and Ganoderma lucidum Higher Basidiomycetes Evidence from Animal Studies. Int J Med Mushrooms. 17(10): 943-55.

67. Han M, Ling MT, Chen J. The Key Role of Mitochondrial Apoptotic Pathway in the Cytotoxic Effect of Mushroom Extracts on Cancer Cells. Crit Rev Eukaryot Gene Expr. 2015; 25(3): 253-8.

68. Doğan HH, Akbaș G. Biological activity and fatty acid composition of Caesar's mushroom. Pharm Biol. 2013 Jul; 51(7): 863-71.

69. Lim JH, Gerhart Hines Z, Dominy JE, et al. Oleic acid stimulates complete oxidation of fatty acids through protein kinase A-dependent activation of SIRT1-PGC1 $\alpha$ complex. J Biol Chem. 2013 Mar 8; 288(10): 7117-26.

70. Guest J, Garg M, Bilgin A, et al. Relationship between central and peripheral fatty acids in humans. Lipids Health Dis. 2013 May 28; 12: 79.

71. Elsayed EA, Enshasy El, Wadaan H, et al. Mushrooms a potential natural source of anti-inflammatory compounds for medical applications. Mediat. Inflamm. 2014; 805841.

72. Enshasy El, Elsayed H, Aziz EA, et al. Mushrooms and truffles: historical biofactories for complementary medicine in Africa and in the Middle East. Evid Based Complement. Altern Med. 2013; 620451.

73. Paterson RR, Lima N. Biomedical effects of mushrooms with emphasis on pure compounds. Biomed J. 2014; 37: 357-368.

74. Komura DL, Ruthes AC, Carbonero ER, et al. 2014. Water-soluble polysaccharides from Pleurotus ostreatus var florida mycelial biomass. Int J Biol Macromol. 2014(Sep); 70: 354-359. http://dx.doi. org/10.1016/ j.ijbiomac.2014.06.007 (Epub 2014 Jul 5).

75. Wasser SP. 2014. Medicinal mushroom science current perspectives advances evidences and challenges. Biomed J. 2014; 37: 345-356.

76. Lindequist U, Kim HW, Tiralongo E, et al. Medicinal mushrooms Evid Based Complement. Alternat Med. 2014; 806180.

77. da Silva AF, Sartori D, Macedo Jr, et al. Effects of b-glucan extracted from Agaricus blazei on the expression of ERCC5 CASP9 and CYP1A1 genes and metabolic profile in HepG2 cells. Hum Exp Toxicol. 2013; 32: 647-654

78. Monro JA. Treatment of cancer with mushroom products. Arch Environ Health. 2003; 58: 533-537.

79. Jeong SC, Koyyalamudi SR, Hughes J, et al. Antioxidant and immunomodulating activities of exo-and endopolysaccharide fractions from submerged mycelia cultures of culinary-medicinal mushrooms. Int J Med Mushrooms. 2013; 15: 251- 266.

80. Xu T, Beelman RB, Lambert JD. The cancer preventive effects of edible mushrooms. Anticancer Agents Med Chem. 2012; 12: 1255-1263. 
81. Walton EL. Buried treasure: unlocking the secrets of medicina mushrooms. Biomed J. 2014; 37 (6): 339-342.

82. Wang J, Liu YM, Cao W, et al. Anti-inflammation and antioxidant effect of cordymin a peptide purified from the medicinal mushroom Cordyceps sinensis in middle cerebral artery occlusion-induced focal cerebral ischemia in rats. Metab Brain Dis. 2012; 27: 159- 165.

83. Wong JH, Ng TB, Wang H, et al. Cordymin an antifungal peptide from the medicinal fungus Cordyceps militaris. Phytomedicine. 2011 Mar 15; 18(5): 387-92.

84. Cui J, Goh KK, Archer R, et al. Characterisation and bioactivity of protein-bound polysaccharides from submerged-culture fermentation of Coriolus versicolor Wr-74 and ATCC-20545 strains. J Ind Microbiol Biotechnol. 2007; 34: 393-402.

85. Cheng KF, Leung PC. General review of polysaccharopeptides PSP from $\mathrm{C}$ versicolor Pharmacological and clinical studies. Cancer Therapy. 2008; Vol 6: 117-130.

86. Cui J, Chisti Y. Polysaccharopeptides of Coriolus versicolor physiological activity uses and production. Biotechnol Adv. $2003 \mathrm{Apr}$; 21(2): 109-22. Review.

87. Trovato A, Siracusa R, Di Paola R, et al. Redox modulation of cellular stress response and lipoxin A4 expression by Hericium Erinaceus in rat brain: relevance to Alzheimer's disease pathogenesis. Immun Ageing. 2016b; 13: 23.

88. Chu KKW, Ho SSS, Chow AHL. Coriolus versicolor A medicinal mushroom with promising immunotherapeutic values. J Clin Pharmacol. 2002.

89. Zhou XW, Hua J, Lin J, et al. Cytotoxic activities of Coriolus versicolor Yunzhi extracts on human liver cancer and breast cancer cell line. Afr J Biotechnol. 2007; 6: 1740-43.
90. Pang ZJ, Chen Y, Zhou M. Polysaccharide Krestin enhances manganese superoxide dismutase activity and mRNA expression in mouse peritoneal macrophages. Am J Chin Med. 2000; 28(3-4): 331-41.

91. Sakagami H, Aoki T, Simpson A, et al. Induction of immunopotentiation activity by a protein-bound polysaccharide PSK review. Anticancer Res. 1991 Mar-Apr; 11(2): 993-9. Review.

92. Yang MM, Chen Z, Kwok JS. The anti-tumor effect of a small polypeptide from Coriolus versicolor SPCV. Am J Chin Med. 1992; 20(3-4): 221-32.

93. Ng TB. A review of research on the protein-bound polysaccharide polysaccharopeptide PSP from the mushroom Coriolus versicolor Basidiomycetes Polyporaceae. Gen Pharmacol. 1998 Jan; 30(1): 1-4. Review

94. Hirahara N, Edamatsu T, Fujieda A, et al. Protein-bound polysaccharide- $\mathrm{K}$ induces apoptosis via mitochondria and p38 mitogen-activated protein kinase-dependent pathways in HL-60 promyelomonocytic leukemia cells. Oncol Rep. 2013 Jul; 30(1): 99104.

95. Ho CY, Kim CF, Leung KN, et al. Coriolus versicolor Yunzhi extract attenuates growth of human leukemia xenografts and induces apoptosis through the mitochondrial pathway. Oncol Rep. 2006 Sep; 16(3): 609-16.

96. Nakamura T, Cho DH, Lipton SA. Redox regulation of protein misfolding, mitochondrial dysfunction synaptic damage and cell death in neurodegenerative diseases. Exp Neurol. 2012 Nov; 238(1): 12-21.

97. Biasibetti R, Tramontina AC, Costa AP, et al. Green tea epigallocatechin3-gallate reverses oxidative stress and reduces acetylcholinesterase activity in a streptozotocin-induced model of dementia. Behav Brain Res. 2013; 236: 186-93. 